\title{
Multiscale 3D Investigation of Damage in Angle-Interlocked Ceramic Matrix Composite under in situ Loading
}

\author{
Aly Badran ${ }^{1}$, Hrishikesh Bale ${ }^{2 *}$, Stephen Kelly ${ }^{2}$, David Marshall ${ }^{1}$ \\ 1. Mechanical Engineering, University of Colorado, Boulder CO, United States. \\ 2. Carl Zeiss X-ray Microscopy, Pleasanton, CA, United States. \\ * Corresponding author: hrishikesh.bale@zeiss.com
}

Textile composites with ceramic fiber tows woven into $2 \mathrm{D} / 3 \mathrm{D}$ architectures in a ceramic matrix are important material options for many structural applications [1]. These composites represent a new class of integrally woven ceramic-matrix-composites for high-temperature applications, where both the strength and thermal conductivity are important. For high-performance and reliability, a key issue is irregularities and defects in the textile reinforcement, which comprise strength and life [2]. Environmental barrier coatings further expand the capabilities for extremely corrosive environments. Due to its strong oxidation resistance and high strength, $\mathrm{SiC}$ is being used in advanced structural applications, but extra processing is required to overcome its inherently brittle behavior. As one route to address this problem, $\mathrm{SiC}$ is processed in the form of composites consisting of complex microstructural architectures derived by weaving bundles of fibers, including functional coatings on the fibers and incorporating several strategies to enhance the toughness of bulk composite.

In recent years, high resolution X-ray imaging capabilities that were previously only available at synchrotron beamlines have been transferred to analogous lab-based instruments; using lab x-ray tubes but incorporating optical elements to achieve the resolution and contrast comparable to many synchrotron experiments, and even adopting an increasing variety of imaging modalities. The lab X-ray tomography systems have benefitted from ongoing development of various types of in situ and 4D imaging experiments, albeit at a different time scale than synchrotrons, presenting both unique challenges as well as opportunities to the researcher.

In this work, we conducted high resolution tomographic imaging of a model CMC system consisting of $\mathrm{SiC}$ fibers in a $\mathrm{SiC}$ matrix. The mini-composite specimen was coated with an environmental barrier coating through repeated cycles of slurry infiltration. Another composite specimen with a woven architecture made up of $\mathrm{SiC} / \mathrm{SiC}$ tows was tested under in-situ tensile loading experiments on a laboratory X-ray Microscope (XRM).

In static 3D X-ray imaging experiments, the reconstructed data from high resolution tomography scans revealed various components of the CMC model specimen with an excellent contrast as seen in Fig. 1. Microstructural features such as fibers, $\mathrm{BN}$ fiber coating, matrix voids, cracks within the matrix and other defects were easily discerned. Additionally, the contrast was high enough to highlight the extent of each individual EBC layer thickness deposited per cycle of the infiltration process. In situ experiments on the other hand revealed the real-time initiation of cracks within tows, and along tow cross-over points as shown in Fig. 2. The images show the process of crack initiation and propagation to form a distributed network of cracks within the composite specimen which imparts the essential toughness in an otherwise brittle material when tested as a monolith. Digital volume correlation (DVC) processing of the 3D tomography datasets revealed the presence of high strain areas in the samples that correspond to sites with prominent cracks. Advanced image processing and segmentation steps were utilized to study the 3D volume of the single tow model composite specimen, where-in a machine learning based 
trainable segmentation approach was used to segment various regions of the composite. The labeled 3D data enabled further quantitative analysis of the composite specimen. The results from the in situ imaging experiment presented here help reveal how cracks initiate and propagate in a CMC at the microstructure level thereby providing insight into the complex fracture behavior.

\section{References:}

[1] DB Marshall, BN Cox, Annu. Rev. Mater. Res. 38 (2008), p. 425.

[2] H Bale et al., Nature Materials 12 (2018).
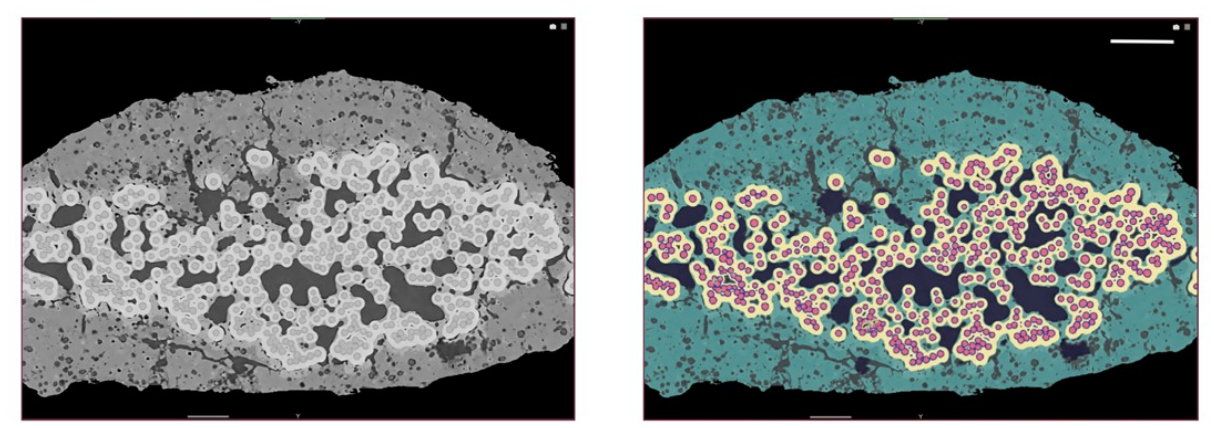

Figure 1. (a) Cross-sectional image of the mini-composite with an EBC layer on the CMC. (b) Segmentation of all the prominent features of the sample. Scale bar $=100 \mu \mathrm{m}$
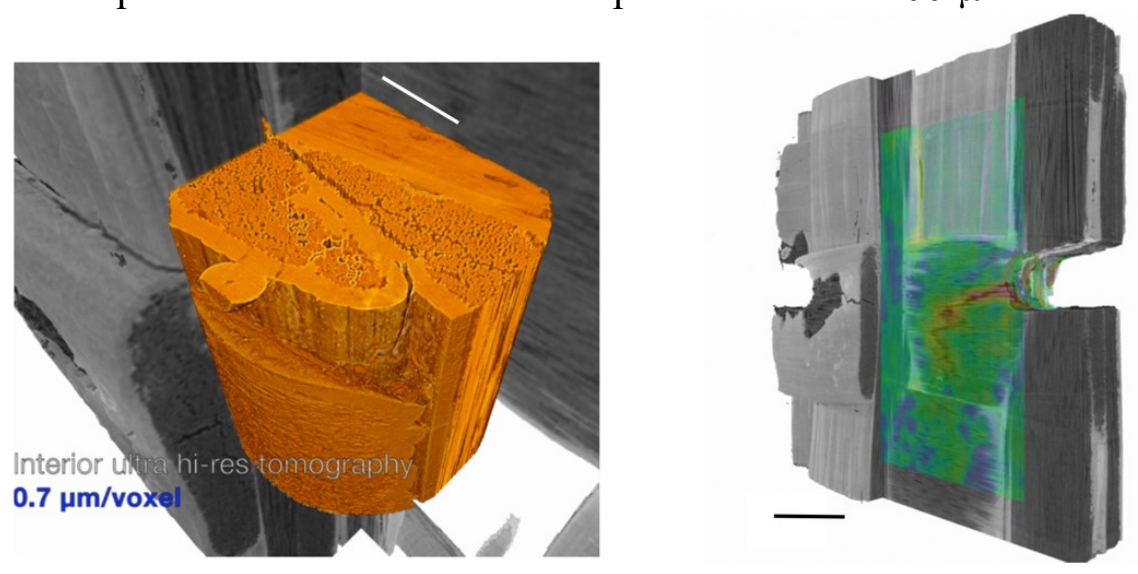

Figure 2. (a) In situ imaging on the Zeiss Xradia 520 Versa XRM under tensile load. Interior volume illustrates high resolution dataset $(0.7 \mu \mathrm{m} / \mathrm{voxel})$ acquired at a large working distance. Scale bar $=100 \mu \mathrm{m}$ (b) DVC reveals strain concentrations in the sample. Scale bar $=1 \mathrm{~mm}$. 